\title{
Dieta e dispersão de sementes por Cerdocyon thous (Linnaeus) (Carnívora, Canidae), em um fragmento florestal no Paraná, Brasil
}

\author{
Vlamir J. Rocha ${ }^{1}$, Nelio R. dos Reis ${ }^{2} \&$ Margareth L. Sekiama ${ }^{3}$ \\ ${ }^{1}$ Klabin S.A., Lagoa, Fazenda Monte Alegre, 84279-000 Telêmaco Borba, Paranaá, Brasil. E-mail: vlamir@klabinpr.com.br \\ 2 Departamento de Biologia Animal e Vegetal, Universidade Estadual de Londrina. 86051-970 Londrina, Paraná, Brasil. \\ ${ }^{3}$ Departamento de Zoologia, Universidade Federal do Paraná. Caixa Postal 19020, 81531-980 Curitiba, Paraná, Brasil.
}

\begin{abstract}
Diet and seed dispersal by Cerdocyon thous (Linnaeus) in a forest fragment in Paraná (Carnivora, Canidae). Although the crab eating fox, Cerdocyon thous (Linnaeus, 1706), is a relatively common Canidae, there isn't much information about its diet and its role as a seed disperser in the different habitats where it occurs. The aim of this work was to report the diet of the $C$. thous and its importance as a seed disperser and $I$ or a seed predator and to test the rate of germination of the seeds after passing through the digestive tract of the animal. The work was carried out in a 680 ha fragment of the Semidecidual Seasonal Forest in the Parque Estadual Mata dos Godoy, located in the city of Londrina-Paraná, south of Brazil. The methodology consisted of the collection of excrement of $C$. thous which were analyzed in laboratory for identification of consumed items and seeds. In germination tests, the seeds were placed to germinate in Petri dishes with wet cotton. Ninety-three animal feces samples, with 219 animal and vegetable items were registered, being $36.52 \%$ remaining portions of small rodents, $24.19 \%$ of grasses, $13.24 \%$ of birds, $10.47 \%$ of insects, $6.39 \%$ of Syagrus romanzoffiana (Cham.) Glassm., $4.6 \%$ of other items of animal origin and $4.54 \%$ of items vegetable origin. In addition, $C$. thous dispersed nine species of plants, with relevant importance to the germination of some seeds, which passed through the digestive tract, except for the most consumed of fruit, $S$. romanzoffiana, whith no seed germination at all in lab conditions. In conclusion, $C$. thous has a generalistc and opportunistc diet, surviving in degraded and anthropic areas and being able to act as a seed disperser.
\end{abstract}

KEY WORDS. Crab-eating fox, frugivory.

RESUMO. Embora o cachorro-do-mato, Cerdocyon thous (Linnaeus, 1706), seja um Canidae relativamente comum, não há muita informação sobre sua dieta e seu papel como dispersor de sementes nos diferentes habitats onde ocorre. O objetivo deste trabalho foi o de reportar a dieta de $C$. thous e sua importância como dispersor e/ou predador de sementes, e ainda testar a taxa de germinação de sementes após passar pelo trato digestório do animal. O estudo foi realizado em um fragmento ( $680 \mathrm{ha}$ ) de Floresta Estacional Semidecidual, o Parque Estadual Mata dos Godoy, localizado na cidade de Londrina, Paraná, sul do Brasil. A metodologia consistiu de coletas de fezes de $C$. thous, as quais foram analisadas em laboratório para identificar os itens consumidos. Nos testes de germinação, as sementes foram dispostas para germinar em placas de Petri com algodão umedecido em água. Noventa e três amostras fecais com 219 itens de origem vegetal e animal foram registradas, sendo $36,52 \%$ contendo restos de pequenos roedores, $24,19 \%$ de gramíneas, $13,24 \%$ de aves, $10,47 \%$ de insetos, $6,39 \%$ de Syagrus romanzoffiana (Cham.) Glassm., 4,6\% de outros itens de origem animal e 4,54\% de outros itens de origem vegetal. Ainda, $C$. thous dispersou nove espécies de plantas, com relevante importância para a germinação de algumas sementes que passaram pelo trato digestório do animal, exceto para S. romanzoffiana, cujas sementes não germinaram nas condições de laboratório. Conclui-se que, $C$. thous apresentou uma dieta generalista e oportunista, sobrevivendo em áreas degradadas e antrópicas, e agindo como dispersor de sementes nestes locais. PALAVRAS CHAVE. Cachorro-do-mato, frugivoria.

Os mamíferos constituem um dos grupos de vertebrados que apresentam variações em seu regime alimentar (Pough et al. 1993). A partir do estudo da dieta desses animais, outras informações podem ser obtidas, como a interação planta-animal.
A evolução das interações permitiu que o mutualismo entre plantas e animais frugívoros atingisse seu clímax nas florestas tropicais, onde aves e mamíferos contribuem com a dispersão de sementes de 50 a $90 \%$ das espécies arbóreas e

Revista Brasileira de Zoologia 21 (4): 871-876, dezembro 2004 
arbustivas, em contrapartida, as plantas fornecem frutos, um importante recurso (Howe \& SMALLWOod 1982, JANZEN 1983a, b, JANSON 1983, HERRERA 1985).

Cerdocyon thous (Linnaeus, 1758) é uma espécie de Canidae, com ocorrência em quase todo o Brasil, exceto em partes da Amazônia (WILSON \& REEDER 1993). É encontrado tanto em áreas de floresta como de campo (LANGguth 1975, BerTa 1982, NowaK 1999). Tem hábito preferencialmentenoturno, desloca-se solitário ou aos pares, por trilhas, bordas de mata e estradas à procura de alimentos (Brady 1979, Berta 1982, Peracchi et al. 2002). Ape sar de ser uma espécie comum no Brasil, pouco se conhece sobre sua dieta, principal mente enfocando as diferentes regiões de sua ocorrência, e seu papel como agente dispersor de sementes.

Desse modo, este trabalho tem por objetivo estudar a dieta de $C$. thous durante as estações do ano, verificar qual seu papel como agente dispersor e/ou predador de sementes, etestar se as sementes que passaram pelo tubo digestório desse canídeo se mantém viáveis.

\section{MATERIAL E MÉTODOS}

\section{Área de estudo}

O Parque Estadual Mata dos Godoy tem uma área de 680 ha (Fig. 1) e situa-se nas coordenadas $23^{\circ} 27^{\prime}$ S e $51^{\circ} 15^{\prime} \mathrm{W}$. Apresenta altitude média de $700 \mathrm{~m}$ e é cortado pelo Trópico de Capricórnio. O clima da região é do tipo cfa: clima subtropical, com temperatura média inferior a $18^{\circ} \mathrm{C}$ no mês mais frio (junho) e temperatura média acima de $22^{\circ} \mathrm{C}$ no mês mais quente (fevereiro). As chuvas se concentram nos meses de verão, contudo não há estação seca definida. O Parque compreende uma ilha de vegetação bem preservada, totalmente circundada por terras cultivadas e por pequenos fragmentos de florestas com diferentes graus de alteração, alguns dos quais interligam-se com o Parque. Segundo M AACK (1981), a área caracteriza-se como Floresta Estacional Semidecidual.

\section{Metodologia}

O período do estudo foi de dois anos, com início em abril de 1996 e término em março de 1998. Foram considerados como estações do ano: verão, os meses de janeiro a março; outono, de abril a junho; inverno, de julho a setembro; primavera, de outubro a dezembro. Para a determinação da dieta e a ação sobre as sementes, foram col etados mensal mente em campo fezes de Cerdocyon thous que eram encontradas em trilhas e bordas da floresta. As fezes foram armazenadas individualmente em sacos plásticos e identificadas com a data da coleta. Posteriormente, em laboratório, as amostras fecais foram triadas com auxílio de microscópio estereoscópico para a identificação dos itens consumidos. As sementes e os fragmentos alimentares encontrados no material fecal foram separados para posterior identificação e testes laboratoriais. A freqüência dos itens alimentares nas fezes foi feita da seguinte forma, para cada amostra fecal, cada item presente, independente da quantidade, foi considerado como um registro de consumo do item examina-

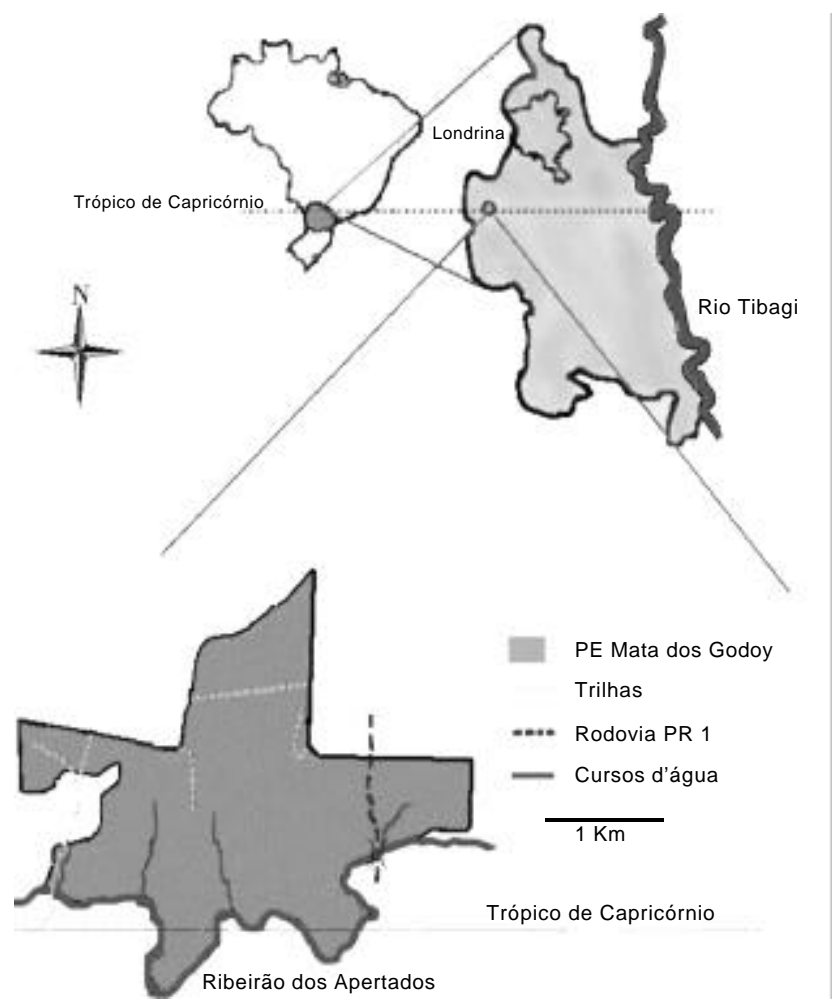

Figura 1. Localização do Parque Estadual Mata dos Godoy (680 ha) no município de Londrina (Paraná).

do. O esforço amostral foi de oito a 12 horas por semana em campo, normalmente realizado em três dias essa variação de horas foi devido à chuvas no período de estudo.

Quanto à ação exercida sobre as sementes, o animal foi considerado dispersor quando depositou fezes contendo sementes em sítios longe da planta mãe e estas passaram intactas pelo tubo digestório; neutro, quando as sementes não foram ingeridas e foram descartadas intactas sob a copa da planta mãe; predador quando as sementes sofreram injúrias por mastigação ou ao passarem pelo tubo digestório do animal.

Para a realização de testes de germinação, sementes encontradas nas fezes foram semeadas em placas de "petri" com al godão umedecido em água e acompanhadas por um período de seis meses (procedimento adaptado de RAmIREZ 1976 e ReIS \& Guillaumet 1983). Para os testes de germinação foram utilizadas 12 sementes de Syagrus romanzoffiana (Cham.) Glassm., 10 de Croton floribundus Spreng. e 16 de Hovenia dulcis Thumb.

\section{RESULTADOS}

Foram coletadas 93 amostras fecais de C. thous, sendo 28 no outono, 25 no inverno, 19 na primavera e 21 no verão, que continham 219 itens alimentares, 142 de origem animal e 77 de origem vegetal (Tabs I e II). 
Tabela I. Alimentos de origem animal, parte encontrada nas fezes, número de vezes que o item foi consumido durante as estações e porcentagem total com que o item participou na dieta de C. thous, no Parque Estadual Mata dos Godoy, Londrina (Paraná), durante o período de abril de 1996 a março de 1998.

\begin{tabular}{|c|c|c|c|c|c|c|c|}
\hline Categoria Taxonômica & Parte Encontrada & Out & Inv & Prim & Ver & $\mathrm{n}$ & $\%$ \\
\hline Araneae & Quelíceras, mandíbulas & & & 1 & 1 & 2 & 0,91 \\
\hline Coleoptera & Carapaça, quitina & 3 & & 4 & 2 & 9 & 4,10 \\
\hline Odonata & Cabeça & & & 1 & & 1 & 0,45 \\
\hline Orthoptera & Patas posteriores & & 3 & & 2 & 5 & 2,28 \\
\hline Hymenoptera & Cabeça & & & 1 & & 1 & 0,45 \\
\hline Insecta & Fragmentos & 3 & & & 4 & 7 & 3,19 \\
\hline Bothrops jararaca (Wied, 1820) & Escamas & & & 1 & & 1 & 0,45 \\
\hline Ophidia & Escamas & & & & 1 & 1 & 0,45 \\
\hline Mammalia & Pêlos e garras & & 1 & 2 & 1 & 4 & 1,80 \\
\hline Mammalia, Rodentia (Sigmodontinae) & Pêlos, dentes, ossos, garras & 26 & 22 & 14 & 18 & 80 & 36,53 \\
\hline Mammalia, Rodentia Sphiggurus villosus (F. Cuvier, 1823) & Pêlos, garras & & & 1 & & 1 & 0,45 \\
\hline Mammalia Xenartrha Dasypus novemcinctus (Linnaeus, 1758) & garras, pedaços de carapaça & 1 & & & & 1 & 0,45 \\
\hline Aves & Penas, pés, bicos, garras e ossos & 10 & 5 & 5 & 9 & 29 & 13,24 \\
\hline Total & & 43 & 31 & 30 & 38 & 142 & 64,75 \\
\hline
\end{tabular}

Tabela II. Espécies vegetais, parte da planta consumida, número de vezes que o item foi consumido durante as estações e porcentagem total com que $\mathrm{o}$ item participou na dieta de $C$. thous. ( $P C=$ Parte consumida) e ação sobre as sementes $(D=$ dispersor, $N=$ neutro, $P=$ predador), no Parque Estadual Mata dos Godoy, Londrina (Paraná), durante o período de abril de 1996 a março de 1998.

\begin{tabular}{|c|c|c|c|c|c|c|c|c|c|}
\hline Família & Espécie & PC & Out & Inv & Prim & Ver & $\mathrm{n}$ & $\%$ & Ação \\
\hline Arecaceae & Syagrus romanzoffiana (Cham.) Glassm. & Frutos & 3 & 3 & & 8 & 14 & 6,39 & $\mathrm{D}$ \\
\hline Euphorbiaceae & Croton floribundus Spreng. & Frutos & 1 & & & & 1 & 0,45 & $\mathrm{D}$ \\
\hline \multirow[t]{5}{*}{ Poaceae } & Brachiaria plantaginea (Link) Hitchc. & Folhas, sementes & 3 & 4 & 3 & 8 & 18 & 8,22 & $\mathrm{D}$ \\
\hline & Panicum maximum Jack* & Folhas, sementes & & 3 & & & 3 & 1,37 & $\mathrm{D}$ \\
\hline & Gramineae & Folhas, sementes & & & & 4 & 4 & 1,82 & $\mathrm{D}$ \\
\hline & Gramineae & Folhas, sementes & 9 & 3 & 8 & 11 & 31 & 12,30 & $\mathrm{D}$ \\
\hline & Zea mays Linnaeus* & Sementes & & 1 & & & 1 & 0,45 & $\mathrm{P}$ \\
\hline Lauraceae & Persea americana Mill* & Frutos & 2 & 1 & & & 3 & 1,37 & $\mathrm{~N}$ \\
\hline Meliaceae & Guarea sp. & Frutos & & 1 & & & 1 & 0,45 & $\mathrm{D}$ \\
\hline Solanaceae & Solanum sp. & Frutos & & & 1 & & 1 & 0,45 & $\mathrm{D}$ \\
\hline Rhamnaceae & Hovenia dulcis Thumb.* & Frutos & 1 & 2 & & & 3 & 1,37 & $\mathrm{D}$ \\
\hline Indeterminada & Indeterminada & Frutos & & 1 & & & 1 & 0,45 & $\mathrm{~N}$ \\
\hline Total & & & 19 & 19 & 12 & 27 & 77 & 35,10 & \\
\hline
\end{tabular}

(*) Espécies exóticas.

Em relação ao total dos itens registrados para a dieta de C. thous, vertebrados foram os itens mais consumidos, principalmente pequenos roedores (Sigmodontinae) com 36,53\% das ocorrências ( $n=80)$, e também aves com 13,24\% ( $n=29)$. Destaca-se também o consumo de duas serpentes, uma Colubridae não identificada e uma Viperidae Bothrops jararaca (Wied,
1820). Quanto aos invertebrados, insetos ocorreram em $10,47 \%$ $(\mathrm{n}=25)($ Tab. $\mathrm{l})$.

Em relação às famílias vegetais, Poaceae predominou com cinco espécies. Já em relação aos itens vegetais, das 12 espécies consumidas, fol has de gramíneas tiveram maior consumo, com $24,16 \%$ das ocorrências ( $n=53$ ), frutos de Syagrus romanzoffiana 
perfizeram 6,39\% ( $n=14$ ), as demais espécies foram consumidas em baixa freqüência (Tab. II).

Em uma observação noturna, foi registrado o comportamento de um par de $C$. thous se alimentando de Persea americana Mill (abacate). Enquanto um indivíduo comia parte da polpa do fruto, o outro ficava em al erta, eassim ocorreu revezamento até consumirem todo o fruto, descartando a casca e semente.

Ositens de origem animal predominaram em relação aos vegetais em todas as estações do ano. No outono, pequenos roedores participaram com $41,23 \%$ das ocorrências $(n=26)$, aves com 16,21\% ( $n=10$ ) e a família Poaceae com 19,34\%. ( $=12$ ). No inverno, pequenos roedores perfizeram $44 \%$ das ocorrências ( $n=22)$, aves $10 \%(n=5)$ e Poaceae $22 \%(n=11)$. Durante a primavera, pequenos roedores foram consumidos com uma freqüência de $33,33 \%(n=14)$, insetos somados perfizeram $14,28 \%$ ( $n=7$ ) e Poaceae $26,18 \%(n=11)$. No verão, pequenos roedores perfizeram $28,57 \%(n=18)$, aves $14,28 \%$ ( $n=9$ ) e Poaceae $26,96 \%$ ( $n=19$ ) (Fig. 2).

Todas as 93 amostras fecais de $C$. thous coletadas, encontravam-se depositadas em bordas da floresta, áreas abertas e uma pequena estrada de $1 \mathrm{~km}$ que cortava a porção norte do Parque e, do total de 12 espécies vegetais registradas em sua dieta, C. thous atuou como dispersor em nove e foi neutro em duas. A categoria predador só foi registrada durante o inverno, para Zea mays Linnaeus (milho). Enquanto a categoria dispersor predominou em todas as estações (Tab. II, Fig. 3).

Quanto aos testes de germinação, as sementes de S. romanzoffiana não germinaram; em Croton floribundus germinaram três sementes corresponden do uma taxa de germinação de $30 \%$, sendo que os dias em que ocorreram germinação foram no $92^{\circ}, 162^{\circ}$ e $182^{\circ}$ dia após o plantio. Quanto a Hovenia dulcis, germinaram nove sementes, correspondendo a 56,25\%, com o maior pico de germinação ocorrendo no 33 dia.

\section{DISCUSSÃ O}

Em relação à dieta, $C$. thous é considerada uma espécie oportunista, sobrevivendo em áreas degradadas e antrópicas (LANGguth 1975, Motta-Júnior et al. 1994, Facure \& MonteiroFILHO 1996). No presente estudo, sua dieta foi baseada em itens de origem animal, principalmente pequenos roedores, aves, insetos, e ainda itens vegetais.

Apesar do registro de 12 espécies vegetais consumidas por C. thous, cinco foram gramíneas (Poaceae), tornando esta família a melhor representada, porém de pouca ou nenhuma importância quanto ao retorno energético. Provavelmente seu consumo esteja relacionado com o auxílio na digestão do animal, corroborando com MotTA-JúnIOR et al. (1994).

As demais espécies tiveram seus frutos consumidos, destacando-se Syagrus romanzoffiana, assim como no estudo de FACURE \& M ONTEIRO-FILHO (1996) em área de vegetação secundária rodeada por pastos e áreas agrícolas na região de Campinas (São Paulo) e também no estudo de MotTA-JúnIor et al. (1994) em áreas de cerrado e de floresta de galeria com grande ação antrópica na região de São Carlos (São Paulo). Em outras áreas dos Estados do Paraná e de São Paulo, sementes dessa espécie de fruto também são observadas com freqüência nas fezes de C. thous.

$\mathrm{Na}$ análise sazonal da dieta de $\mathrm{C}$. thous, quanto aos itens de origem animal, foi constatado que pequenos roedores eaves foram os mais freqüentemente consumidos em todas as estações. O alto consumo destes recursos está relacionado com a abundância desses grupos na área de estudo, pois muitas das áreas vizinhas ao Parque são de cultivo de soja, trigo e milho, os quais servem de fonte al imentar para pequenos roedores e aves. Esses dados corroboram com os de M ONDOLFI (apud W ALKER 1975), que registrou pequenos roedores como os itens mais abundantes em 19 conteúdos estomacais de $C$. thous, o que coloca a espécie como um importante agente controlador da população de pequenos roedores na área de estudo, fato este também citado por BISBAL \& OJASTI (1980). Nos Ihanos venezuelanos, BRADY (1979) relata uma alta porcentagem de vertebrados na dieta de $\mathrm{C}$. thous (constituída de lagartos, cobras e roedores), principalmente na estação seca. Porém, outros autores relatam um maior consumo de frutos (BISBAL \& OJAsti 1980, Motta-Júnior et al. 1994, FACure \& Monteiro-Filho 1996, MacDonald \& Courtenay 1996). Provavelmente dietas diferenciadas ocorrem de acordo com a disponibilidade de recursos em regiões diferentes.

Em relação ao consumo de Bothrops jararaca, os canídeos, ao contrário dos felinos (que eventualmente consomem serpentes) (obs. pes.), não são tão ágeis na caça desses animais, o que poderia oferecer certo risco. Presume-se que $C$. thous possa ter al guma estratégia comportamental diferenciada para a captura de ofídios peçon hentos ainda não observada ou provavelmente ter encontrado a serpente morta, e a ingerido. O consumo de animais mortos por $C$. thous já foi registrado por outros autores (MotTA-Júnior et al. 1994, FACURE \& M ONTEIRO-FILHo 1996).

Em relação à observação do casal de $C$. thous se alimentando alternadamente de Persea americana, fica claro o comportamento cooperativo que a espécie pode apresentar em certas ocasiões. Nowak (1999) e Emmons \& Feer (1997) afirmam que, apesar desses animais viajarem juntos, cada indivíduo captura seu próprio alimento. Todavia, Brady (1979) também observou a cooperação entre casal durante a caça.

Quanto à dispersão de sementes por C. thous, das nove espécies que foram consumidas, quatro eram gramíneas com baixo potencial de dispersão zoocórica, já que são espécies anemocóricas (VAN DER PIJL 1982). As demais sementes eram de espécies arbóreas, sendo importante para as mesmas o fato de terem suas sementes dispersadas para longe da planta-mãe. Dentre as espécies arbóreas, sementes de Syagrus romanzoffiana foram as mais dispersadas, devido ao grande consumo. Porém, apesar de $C$. thous dispersar as sementes dessas plantas, aparentemente sua efetividade no processo de recrutamento de novas plântulas é questionável, pois as fezes que continham 

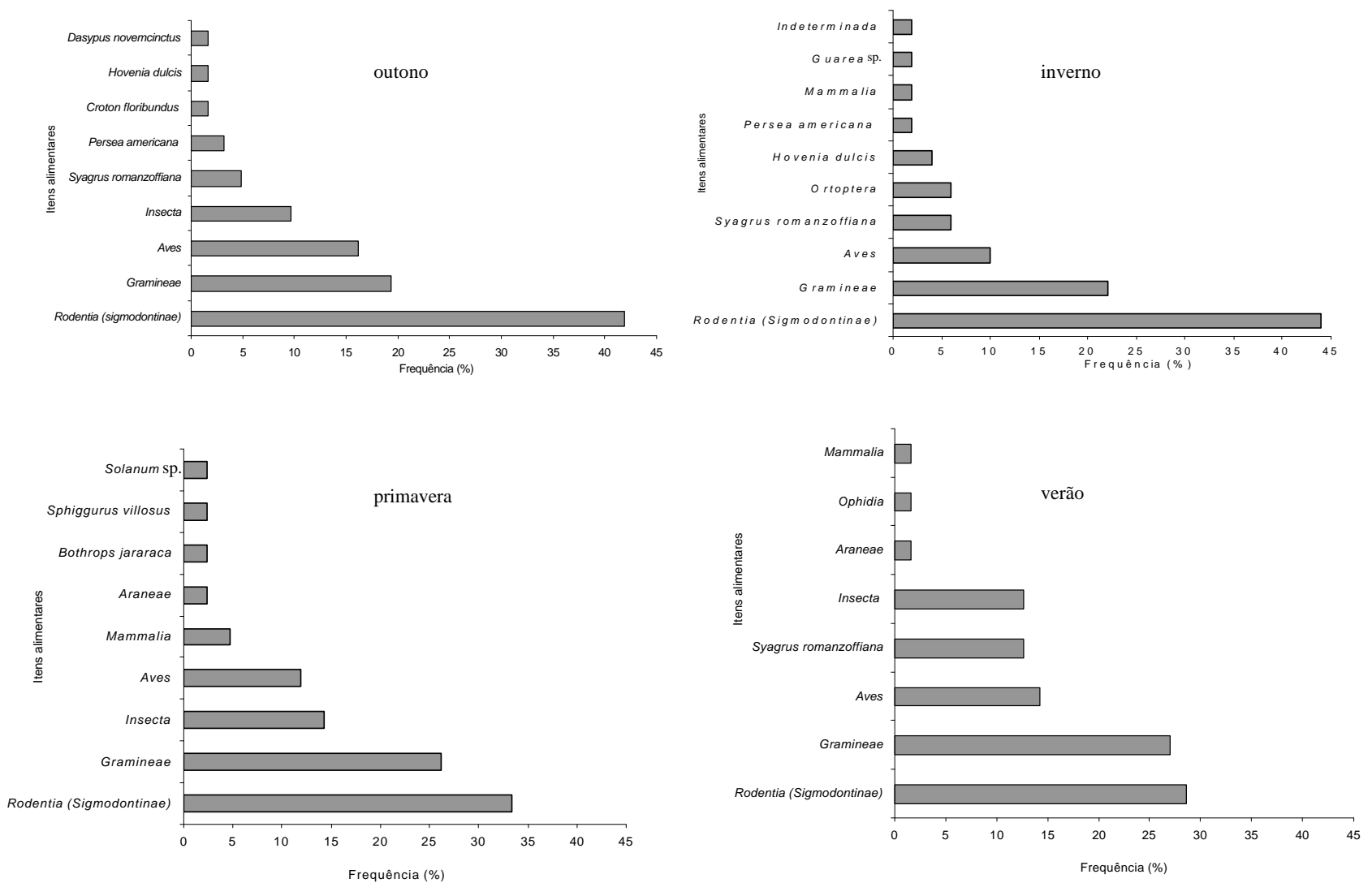

Figura 2. Freqüência de ocorrência (\%) com que diferentes itens foram consumidos durante as estações do ano por C. thous, no Parque Estadual Mata dos Godoy, Londrina (Paraná), durante o período de abril de 1996 a março de 1998.

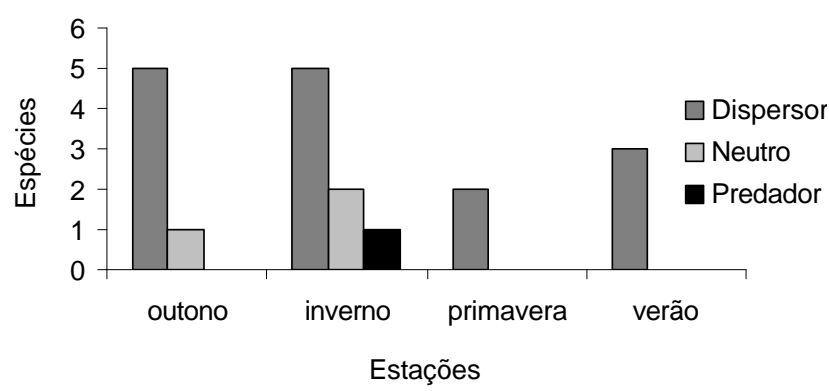

Figura 3. Ação de C. thous sobre as sementes durante as estações do ano, no Parque Estadual Mata dos Godoy, Londrina (Paraná), durante o período de abril de 1996 a março de 1998.

sementes se encontravam em locais desfavoráveis à germinação e ao estabelecimento de novas plântulas, como bordas de floresta, áreas abertas e estradas, expostas a fatores ambientais negativos como alta radiação solar, baixa umidade e compactação do solo. Bustamante et al. (1992), trabal hando com Pseudalopex culpaeus (Molina,1782), também relatam que esse canídeo, apesar de ser um bom dispersor, defeca em áreas abertas, desfavoráveis à germinação. Mesmo que essas sementes germinassem nessas áreas, as plântulas ficariam sujeitas a predadores e à desidratação. No presente estudo, em nenhuma ocasião foi observado qualquer tipo de semente germinando nas fezes de C. thous, todavia Alonso-PAz et al. (1995) em áreas de planícies abertas no Uruguai, encontraram em três fezes diferentes sementes da palmeira butia (Butia capitata) germinando na natureza.

O fato de poucas espécies serem dispersas por $C$. thous na área de estudo, está diretamente relacionado com sua dieta, que se baseou em itens de origem animal, principalmente pequenos roedores. Em outras localidades dos estados do Paraná e de São Paulo, C. thous parece ser um importante agente dispersor, pois suas fezes contêm grande quantidade de sementes intactas de espécies arbustivas e arbóreas (obs. pes.). No entanto, são necessários mais estudos para confirmar sua efetividade como agente dispersor. MOTTA-JúNIOR et al. (1994), trabal hando em área de cerrado e em floresta de gal eria, relataram um total de quatorze espécies dispersadas por esse animal, e Alonso-PAz et al. (1995) encontraram em áreas de planícies 
abertas no Uruguai grande quantidade de sementes de Butia capitata, dispersadas através das fezes, o que demonstra que $C$. thous pode ser um importante agente dispersor de sementes.

Nos testes de germinação, C. thous foi eficaz para al gumas espécies, como para Hovenia dulcis, na qual mais de $50 \%$ das sementes germinaram. Para Croton floribundus ocorreu $30 \%$ de germinação, que pode ser considerada uma taxa bem expressiva, uma vez que essa éuma espécie que possui frutos autocóricos, os quais não apresentam muitos atrativos para serem consumidos por mamíferos. Todavia, ambas as espécies levaram muito tempo para germinar nas condições de laboratório. Quanto a Syagrus romanzoffiana, quefoi a espécie de fruto mais consumida, nos testes de laboratório nenhuma semente germinou, o que pode ter relação com as condições durante os experimentos, as quais não tenham sido ideais para que as sementes germinassem. MotTA-JúnIor et al. (1994) testaram, em laboratório, a germinação de sementes de 14 espécies defecadas por $C$. thous, e verificaram que sete espécies germinaram (em taxas que variaram entre 49,1 e 100\%), e em sete não ocorreu germinação.

\section{REFERÊNCIAS BIBLIOGRÁ FICAS}

Alonso-Paz, E.; R. Rodriguez-Mazzini \& M. Clara. 1995. Dispersión dela “Palma Butiá" (Butia capitata) por el "zorro de monte" (Cerdocyon thous) en montes nativos de la Reserva deBiósfera Bañados del Este, Uruguay. Comunicaciones Botánicas del Museo de Historia Natural de Montevideo, Montevideo, 104: 1-4.

BISBAL, F.J. \& J. OJASTI. 1980. Nicho trofico del zorro Cerdocyon thous (Mammalia, Carnivora). Acta Biologica Venezuelica, Caracas, 10 (4): 469-496.

Berta, A. 1982. Cerdocyon thous. Mammalian Species, Washington, 186: 1-4.

BradY, C.A. 1979. Observations on the behavior and ecology of thecrab-eating fox (Cerdocyon thous). p.161-167. In:J.F. EISENBERG (Ed.). Vertebrate ecology in the Northern Neotropics. Washington, D.C., Smithsonian Institution Press, 271p.

Bustamante, R.O.; J.A. Simonetti \& J. E. Mella. 1992. Are fox legitimate and efficient seed dispersers? A field test. Acta Oecologica, Montrouge, 13 (2): 203-208.

Emmons, L.H. \& F. Feer. 1997. Neotropical rainforest mammals. A field guide. Chicago, University of Chicago Press, $2^{\text {nd }}$ ed., $X V I+307 p$.

Facure, K.G. \& E.L.A. Monteiro-Filho. 1996. Feeding habits of the Crab-eating fox, Cerdocyon thous (Carnivora, Canidae), in a suburban area of southeastern Brazil. Mammalia, Paris, 60 (1): 147-149.

HerRera, C.M. 1985. Determinants of plant-animal coevolution: the case of mutualistic dispersal of seed by vertebrates. Oikos, Lund, 44: 132-141.

Recebido em 01.IV.2004; aceito em 06.XI.2004.

Revista Brasileira de Zoologia 21(4): 871-876, dezembro 2004
Howe, H.F. \& J.S. Smallwood. 1982. Ecology of seed dispersal. Annual Review of Ecology and Systematics, Palo Alto, 13: 201-223.

JANSON, C.H. 1983. Adaptation of fruit morphology to dispersal agents in a neotropical forest. Science, New York, 219: 187189.

JANZEN, D.H. 1983a. Dispersal of seeds by vertebrate guts, $p$. 232-262. In. D.J. Futuyma \& M. SlatKIn (Eds). Coevolution. Sunderland, Sinauer Associates Inc., $9^{\text {th }}$ ed., $X+555 p$.

- 1983b. Seed and pollen dispersal by animals: convergence in the ecology of contamination and sloppy harvest. Biological Journal of the Linnean Society, London, 20: 103-113.

LANGGUTH, A. 1975. Ecology and evolution in the South American canids, p. 192-206. In: M.W. Fox (Ed.). The wild canids: their systematics, behavioral ecology, and evolution. New York, van Nostrand Reinhold Co., XVI+508p.

MAACK, R. 1981. Geografia física do Estado do Paraná. Rio de Janeiro, J. Olympio, 2 ${ }^{\mathrm{a}}$ ed. XLIII+450p.

MaCDonald, D.W. \& O. CouRTenay. 1996. Enduring social relationships in a population of crab-eating zorros, Cerdocyon thous, in Amazonian Brazil (Carnivora, Canidae). Journal of Zoology, London, 239: 329-355.

MotTA-JúnIOR, J.C.; J.A Lombardi \& S.A. TALAmoni. 1994. Notes on crab-eating fox (Dusicyon thous) seed dispersal and food habits in southeastern Brazil. Mammalia, Paris, 58: 156-159.

NowAK, R.M. 1999. Walker's mammals of the world. Baltimore, The Johns Hopkins University Press, vol. 1, $6^{\text {th }}$ ed., LI +836p.

Peracchi, A.L.; V.J. Rocha \& N.R. Dos ReIs. 2002. Mamíferos não voadores da bacia do rio Tibagi. P.225-249. In: M.E. MEDRI; E. Bianchini; J.A. Pimenta \& O. Shibatta (Eds). A Bacia do Rio Tibagi. Londrina, MC Gráfica, 593p.

VAN DER PIJL, L. 1982. Principles of dispersal in higher plants. Berlin, Springer-Verlag, $3^{\text {rd }}$ ed., $X+214 p$.

Pough, F.H.; J.B. HeISer \& W.N. McFarLand. 1993. A vida dos vertebrados. São-Paulo, Atheneu, 839p.

RAMIREZ, B.W. 1976. Germination of seeds of New World Urostigma (Ficus) and of M orus subra L. (Moraceae). Revista de Biologia Tropical, San José, 24 (1): 1-6.

Reis, N.R. dos \& J.L. Guillaumet. 1983. Les chauves-souris frugivores de la région de $M$ anaus et leur rôle dans la dissémination des espéces végétales. Revue d'Ecologie (Ia Terre et la Vie), Paris, 38: 147-169.

W ALKER, E.P. 1975. Mammals of the world. Baltimore, The Johns Hopkins University Press, Mariland, vol. 2, $3^{\text {rd }}$ ed., 1500p.

Wilson, D.E. \& D.M. Reeder. 1993. Mammal species of the world. A taxonomic and geographic reference. Washington, D.C., Smithsonian Institution Press, The American Society of Mammalogists, $2^{\text {nd }}$ ed., 1206p. 